\title{
Report from the "Who Decides?” Workgroup
}

\author{
Ivy Anderson, Mel DeSart, Lee Cheng Ean, Remi Gaillard, Susan Gibbons, Adam Huftalen, Joan \\ Lippincott, Salvatore Mele, Ralf Schimmer, Deborah Stine, John Vaughn
}

\begin{abstract}
Who decides the future of open access, or, rather, who has the power to make decisions that can affect the future of open access? We believe that large scale, transformative, and inclusive progress on these questions can transpire when several entities, each with different complementary powers, convene to collaborate on win-win solutions. We offer three examples of such possible scenarios: the way scholars are evaluated, the way some innovations in scholarly publishing can be nurtured, and the way global cooperation can transform existing journals to open access.
\end{abstract}

\section{OSI2016 Workgroup Question}

Tied to [the] question of who should decide the future of open access, who should have the power to make changes to scholarly publishing practices? Do these powers flow from publishers, institutions, tenure committees, funding agencies, authors, or all of the above? All of the above? None of the above? What are the pros, cons, and consequences of different institutions and interest groups developing and implementing their own solutions (even the oneoff variety)? Is federal oversight needed? Global coordination (through an organization like UNESCO)?

The question of "who decides" can only be meaningfully understood in the context of particular actions: who decides what? Therefore we consider in this paper not only who has the power to make various decisions, but the kinds of actions or decisions that can produce beneficial, largescale change within scholarly publishing, and which actors are best positioned to take those actions.

The actions we propose below are informed by two overarching goals:

- To enable an economically feasible, sustainable move to open access (OA), while preserving quality and ensuring preservation and access

- To improve the creation and dissemination of new knowledge.

Among several possibilities, we focus on three different proposals supporting these dual goals, which offer the opportunity of immediate concerted action and transformative results:

1. Evaluation: re-assessing criteria for academic tenure and promotion

2. Incubation: nurturing alternative, community-driven publishing models

C 2016 OSI2016 "Who Decides?” Workgroup. This open access article is distributed under the Creative Commons Attribution 4.0 International License. This document reflects the combined input of the authors listed here (in alphabetical order by last name) as well as contributions from other OSI2016 delegates. The findings and recommendations expressed herein do not necessarily reflect the opinions of the individual authors listed here, nor their agencies, trustees, officers, or staff. 
3. Transformation: facilitating a "global flip" of research journals from subscription-based to OA.

Before tackling these proposals, we need to understand the actors and the ways in which their actions can work to effect change.

\section{Stakeholders and their power}

Our group began by identifying the key stakeholders that have power to act in specific ways within the scholarly communication ecosystem-either as going concerns, or as actors of change. We articulated the nature of each stakeholder's power, and therefore the kind of actions it can take (see Figure 1, below). These stakeholders include:
- Funding agencies, including, for example, government and nongovernment entities, have the power of allocating resources and the power to define policies.

- Libraries have spending power, as those who procure information resources, as well as the power of choice-that is, the ability to choose what to invest in.

- Universities have the power of policymaking and power of allocating their resources.

- Publishers, including learned societies, have the power emanating from their ownership of journals and the related publishing infrastructure.

- Researchers, of course, have the power of choosing what and where to publish.

\section{Figure 1: Stakeholders in scholarly communication and their sources of power}

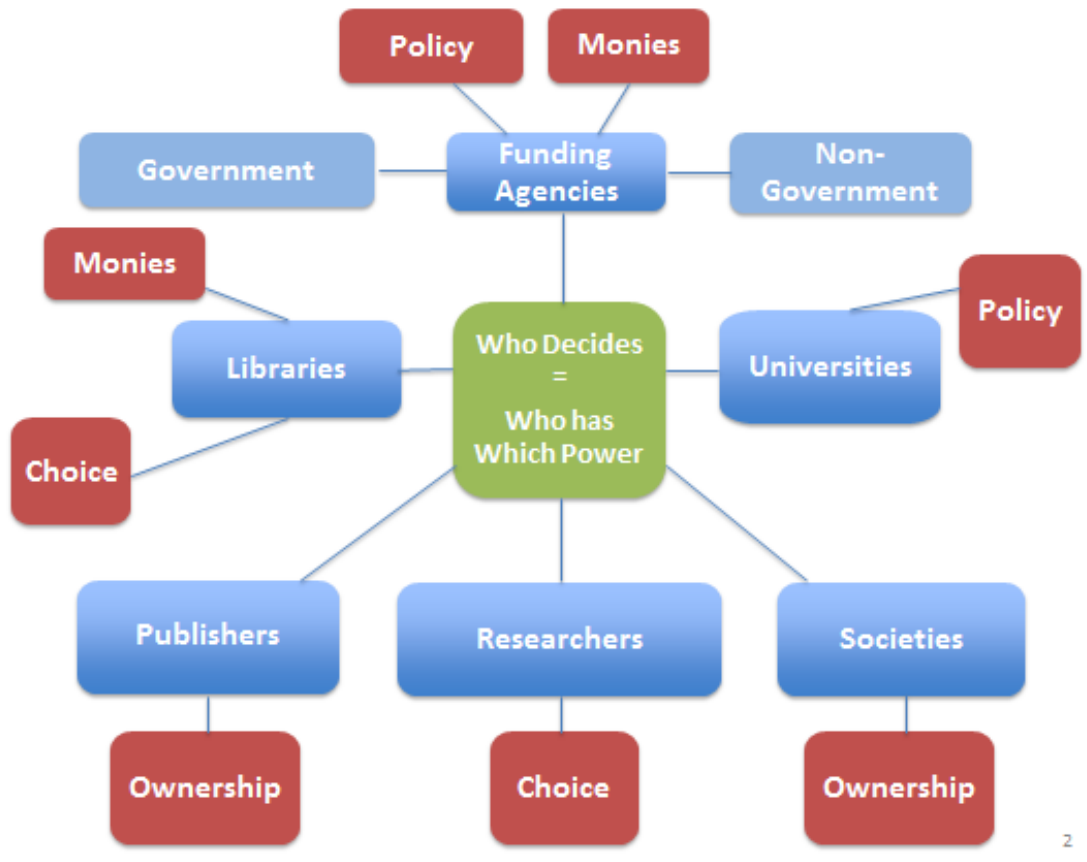


While the public is also an important stakeholder in scholarly communication, as the ultimate beneficiary of public investment in research, and may already have a participatory or advocacy role in various initiatives - particularly those involving the desirability of public access-its ability to directly exercise power appeared to us less direct than the actors mentioned above, with less immediate effect on decisionmaking, thus we did not investigate its impact in depth.

\section{The power to convene}

In order for multiple stakeholders to cooperate in any significant and intentional act of transformation, a convening authority is generally needed to bring the stakeholders together. Examples of organizations in our community with the kind of legitimacy that gives them power to convene include:

- Professional associations-for example, associations of libraries, universities or learned societies, at a national or even a global level

\section{- Funders}

\section{- Inter-governmental organizations}

Independent organizations can also seek to exercise a convening power if they have sufficient stature within a particular community. Indeed, many innovative initiatives may emerge from within particular communities, which then attract additional, external support.

\section{$\underline{\text { PROPOSAL } 1}$}

Evaluation: reviewing research output and assessment criteria

It is widely believed that the manner in which research outputs are evaluated for promotion and tenure as well as for other research assessment exercises can be enhanced to fully value the myriad ways scholars operate and communicate today. Consider, for example, the impact of publishing a curated dataset that allows hundreds of new works to be derived!

The current reliance on journal brand and its associated prestige, often linked to impact factors, is not only an imprecise measure of the quality of a given output, but in some cases may also act as a conservative force creating a bias against newer publishing modalities and impeding change in scholarly practices. In addition, evaluation standards are only beginning to be established for emerging forms of scholarship, such as in the practice of digital humanities. Finally, research evaluation policies can also serve as a lever to encourage more open scholarship.

To further these goals, universities and research communities-by which we mean both learned societies and individual researchers-might be convened by their professional associations to create new evaluation criteria that:

- Fully consider OA publications on the same footing as all other outlets in research assessment, including tenure and promotion decisions

- Research and validate the use of alternative metrics, including both article-level metrics as well as metrics for evaluating emerging forms of research output (examples of these 
include the MLA Guidelines for Evaluating Work in Digital Humanities, ${ }^{1}$ and the San Francisco Declaration on Research Assessment (DORA) Principles ${ }^{2}$ )

- Reward greater openness, such as making research data open, and making versions of articles submitted for evaluation openly available-for example, by leveraging deposit in OA repositories.

\section{How might such an initiative go for- ward?}

This revision of evaluation criteria would be best conducted on a national rather than international scale, as research evaluation frameworks vary from country to country.

In Europe, at the national scale, research organizations like French National Center for Scientific Research-whose ethics committee recently recommended that data sharing be recognized in the assessment and promotion of researchers-can play an important role in promoting and incorporating new criteria into evaluations.

In the US, major national university associations such as the Association of American Universities (AAU) and the Association of Public and Land-grant Universities (APLU) are ideal organizations to convene such a process. Acting individually or jointly, these organizations could convene a range of key stakeholders to discuss options for enhancing the criteria for research evaluation.

These organizations could then charge a multi-stakeholder task force to conduct an environmental scan of relevant initiatives-guidelines, alternative metrics, or policies - and propose a roadmap for further action.

\section{PROPOSAL 2}

Incubation: nurturing alternative publication models

Our second proposal is to cultivate alternative models of publication. These can include lower-cost dissemination platforms for traditional research publication or more transformative initiatives that serve as an outlet for newer types of research.

In this case, libraries as financial contributors; publishers (including, but not limited to, society publishers) as owners of intellectual property and as owners of service provision infrastructure; and research communities as knowledge creators, who exercise choice in disseminating their scholarly work, come together to develop, support, and utilize alternative modes of dissemination (possibly operating at lower cost than legacy models). This includes incubating new models to publish and disseminate content that is not presently part of the scholarly communication ecosystem. These models may be most likely to emerge through independent action of interested stakeholders, often with a disciplinary focus. For example:

- The Online Library of the Humanities $(\mathrm{OLH})$, which is providing an efficient, low-cost platform for open access journals, with funding provided by libraries ${ }^{3}$

- LingOA, an initiative by the linguistics community to transition linguistics journals to the OLH platform ${ }^{4}$

- Libraria, a collective of social science journals and learned societies that have teamed with the Public Knowledge Project to explore a cooperative funding model. ${ }^{5}$ 


\section{How might such an initiative move forward?}

As the above examples suggest, alternative publishing initiatives are most likely to emerge through the self-directed efforts of communities of shared interest. With this setting, libraries can nurture these developments by setting aside funds specifically to invest in such efforts and funding agencies might also provide needed startup capital. Library consortia can also play a convening role by helping to promote such initiatives, offering a vehicle by which to reach large numbers of libraries.

The Open Access Network project is another effort to develop a shared funding capability across libraries and other stakeholders in order to direct needed capital to promising emerging initiatives. ${ }^{6}$

\section{PROPOSAL 3}

Transformation: a "global flip" of research journals to open access

Our third proposal is a global flip of existing subscription journals to open access. In this model, libraries, publishers, and funders, convened by an organization with global standing, come together to redirect subscription funding toward transforming existing journals to open access publication. The idea is to serve the public good and the commons of information, by reusing the same funds that today are spent to provide access to a limited audience. Among the current examples are:

- $\mathrm{SCOAP}^{3}$, which has established a global funding consortium of libraries and research funders under the auspices of CERN to convert a significant portion of the literature of high-energy physics to open access, at no burden to authors, at a cost-per-article considerably lower than existing open access "APC" arrangements"

- A number of publishers and nationallevel library consortia in Europe are developing so-called offsetting pilot agreements in which as part of the contracts to purchase access from providers to a portfolio of journals, and without significant additional cost, an institution's article output is published as open access.

- The OA2020 initiative, recently launched by the Max Planck Society, is soliciting formal "expressions of interest" whereby libraries (on an individual or, preferably, national level) can publicly declare their intention to migrate from subscriptions to open access. ${ }^{8}$ A likely mechanism for this is the offsetting model described above, although other methods could be explored, including combining offsetting with the cultivation of alternative models.

\section{How might such an initiative move forward?}

OA2020 offers an example in which an individual institution has independently put itself forward as a convening authority for a major global initiative of this nature. Other organizations, however, could likewise convene or provide a framework for such a project, particularly organizations with global reach, such as the nascent Global Network of Research-Intensive Universities.

Such an organization might convene a meeting of interested stakeholders to formulate an initiative that would transform subscriptions to open access (as CERN, an intergovernmental organization, did at the beginning of SCOAP3). Institutions and other stakeholders would be invited to 
pledge their interest in order to build support and critical mass.

The initiative could also offer an analytical capability to analyze subscription expenditures and publishing output to help institutions assess the feasibility of such a move. Examples of this already exist: The Max Planck Digital Library is offering such a capability to other interested institutions as part of the OA2020 project, and the University of California's "Pay It Forward" project is also developing tools to facilitate this analysis. ${ }^{9}$ Armed with this information, libraries and library consortia, preferably on a national level, could then explore offsetting arrangements with major publishers, with a goal of full conversion to open access over a defined period of time. During the transition period, libraries and publishers would work together to outline migration paths and develop best practices for this transition to open access. It would behoove these various stakeholders to come together periodically, perhaps annually, under the auspices of the convening body, to assess progress and chart future developments.

\section{Conclusion}

For these transformations to take place, stakeholders and others who have the power to make changes need to come together. To enable access and sharing of open publications and data, funding agencies, universities, libraries and researchers need to collaborate and work toward a common understanding that open scholarship is a necessity.

It is timely for key players to convene meetings and initiatives to transform scholarly communication: Those who have the power to decide can convene to transform and modernize the research evaluation process; empower innovative alternative publishing models; and enable a global flip of the research literature to Open Access.

\section{OSI2016 “Who Decides?” Workgroup}

Ivy Anderson, Interim Executive Director and Director of Collections, California Digital Library (CDL)

Mel DeSart, Head, Engineering Library, University of Washington

Lee Cheng Ean, University Librarian, National University of Singapore

Remi Gaillard, Head of Collection Management Department, University of Pierre and Marie Curie

Susan Gibbons, Deputy Provost, Libraries \& Scholarly Communication, Yale University

Adam Huftalen, Senior Manager of Federal Government Affairs, RELX Group

Joan Lippincott, Associate Executive Director, Coalition for Networked Information (CNI) 
Salvatore Mele, Head of Open Access, European Organization for Nuclear Research (CERN)

Ralf Schimmer, Director, Department of Scientific Information Provision, Max Planck Digital Library, Max Planck Society

Deborah Stine, Professor of the Practice, Engineering and Public Policy, Carnegie Mellon University

John Vaughn, Senior Fellow, Association of American Universities (AAU)

\section{Notes:}

${ }^{1}$ Modern Language Association, "Guidelines for Evaluating Work in Digital Humanities," 2012, as of June 15, 2016: https://www.mla.org/About-Us/Governance/Committees/Committee-Listings/Professional-Issues/Committee-on-InformationTechnology/Guidelines-for-Evaluating-Work-in-Digital-Humanities-and-Digital-Media 2 San Francisco Declaration on Research Assessment (DORA), as of June 15, 2016: http://www.ascb.org/dora/

${ }^{3}$ Online Library of the Humanities (OLH), as of June 15, 2016: https://www.openlibhums.org/

${ }^{4}$ LingOA, as of June 15, 2016: www.lingoa.eu/

${ }^{5}$ Libraria, as of June 15, 2016: http:/ /libraria.cc/

6 Open Access Network, as of June 15, 2016: http:/ /openaccessnetwork.org/

7 SCOAP 3 , as of June 15, 2016: https:/ / scoap3.org/

8 OA2020, as of June 15, 2016: http:/ /oa2020.org/

${ }^{9}$ University of California Libraries, UC Davis, and California Digital Library, "Pay It Forward: Investigating a Sustainable Model of Open Access Article Processing Charges for Large North American Research Institutions," 2014, as of June 15, 2016: http:/ /icis.ucdavis.edu/wp-content/uploads/2014/06/UC-Pay-It-Forward-narrative-2014-FINAL.pdf 